\title{
Manhattan Distance Method
}

National Cancer Institute

\section{Source}

National Cancer Institute. Manhattan Distance Method. NCI Thesaurus. Code C72664.

The sum of the absolute differences between two variables (e.g. Abs[A-B]). Unlike the euclidean distance this does not give an overemphasis to greater distances. This should generally be considered the default distance measure to be used in most operations. 